\title{
Hypofractionated radiotherapy does not increase toxicity in women treated with oncoplastic breast conservation surgery: Initial clinical experience at University of Chicago
}

\author{
Mutlay Sayan ${ }^{1, *}$, Greg Hubert², Julie E. Park ${ }^{3}$, Steven J. Chmura² and Yasmin Hasan² \\ 1 University of Vermont, College of Medicine, Burlington, Vermont, 05405, USA \\ 2 Department of Radiation and Cellular Oncology, University of Chicago, Chicago, Illinois, 60637, USA \\ ${ }^{3}$ Department of Surgery, University of Chicago, Chicago, Illinois, 60637, USA.
}

\begin{abstract}
Oncoplastic breast conservation surgery decreases re-excision procedures and produces desired cosmetic results in patients undergoing breast conservation therapy; however the toxicity of hypofractionated whole breast radiotherapy (HypoRT) has not been previously described in these patients. In this study, we compared the acute and late toxicity of HypoRT to conventionally fractionated radiotherapy (ConvRT) in women treated with oncoplastic breast conservation surgery. Women treated with oncoplastic breast conservation surgery followed by HypoRT or ConvRT from January 2008 through December 2014 were included. Thirty seven patients treated to 38 breasts were identified. Twenty eight were treated with ConvRT and the remainder with HypoRT. Median follow-up was 15.3 months. Grade $\geq 2$ acute toxicity was seen in $50 \%$ and $10 \%$ of the ConvRT and HypoRT patients, respectively $(P=0.0314)$. Grade 3 acute toxicity was reported in 2 (7\%) patients treated with ConvRT and none with HypoRT. The mean breast volume and treatment position were not associated with increased acute toxicity. Skin pigmentation was the most commonly reported late adverse event, noted by $57 \%$ of the ConvRT and $40 \%$ of the HypoRT patients $(P=0.163)$. Two $(7 \%)$ patients treated with ConvRT had lymphedema versus none in HypoRT group. HypoRT is not associated with increased acute and late toxicity among women treated with oncoplastic breast conservation surgery compared to ConvRT in our initial clinical experience. HypoRT can be further explored as a treatment option among this patient population.
\end{abstract}

Keywords: breast cancer; oncoplastic surgery; hypofractionated radiotherapy

\section{Introduction}

Breast conserving therapy comprises breast conservation surgery followed by adjuvant radiotherapy and is standard of care for women with early stage breast cancer. Institutional skin toxicity reporting was used during the treatment and follow-up to score toxicity for each treated breast [1]. Several randomized trials, with up to 20 years of follow-up, have confirmed that breast conserving therapy is equally efficacious as mastectomy with no significant difference in breast cancer mortality and overall survival $[2,3]$. Traditional breast conservation surgery, consisting of local excision of the tumor, has evolved significantly over the years, trending towards procedures that allow complete resection of tumors while preserving cosmesis. Oncoplastic breast surgery was initially described by Audretsch et al. as a local tissue rearrangement during breast conservation surgery [4]. It combines the breast conservation surgery with techniques of plastic surgery to allow clear resection margin while preserving the cosmesis, which be challenging depending on the quadrant of the involved breast. Since then the term "oncoplastic breast surgery" has been used broadly and includes various techniques such as simple glandular flaps, mammoplasties, fasciocutaneous flaps, or myocutaneous flaps [5-7]. Oncoplastic breast surgery is indicated in many cases such as a large tumor to breast size, when conventional surgery opposes major aesthetic sequelae, potential risk of re-excision due to margin involvement, extensive DCIS, invasive lobular carcinoma, multifocal diseases and partial or poor responses to neoadjuvant chemotherapy [8].

The objective of adjuvant radiation therapy following breast conservation surgery is to eradicate any remaining microscopic diseases. Historically, conventionally fractionated radiotherapy (ConvRT) has been the most widely applied radiation scheme which targets the entire

*Corresponding author: Mutlay Sayan, University of Vermont, College of Medicine, 89 Beaumont Ave, Burlington, VT 05405, USA. Tel.: 1-802-8256006; Fax: 1-802-656-8577; Email: mutlay.sayan@med.uvm.edu

Received 05 May 2016 Revised 15 July 2016 Accepted 22 July 2016 Published 28 July 2016

Citation: Sayan M, Hubert G, Chmura SJ, Hasan Y. Hypofractionated radiotherapy does not increase toxicity in women treated with oncoplastic breast conservation surgery: Initial clinical experience at University of Chicago. J Clin Radiat Oncol. 2016; 1(2):7-12. DOI: 10.14312/23978511.2016-2

Copyright: (C) 2016 Sayan M, et al. Published by NobleResearch Publishers This is an open-access article distributed under the terms of the Creative Commons Attribution License, which permits unrestricted use, distribution and reproduction in any medium, provided the original author and source are credited. 
breast with 1.8-2.0Gy per fraction for a total dose of 4550 Gy in 25-28 daily fractions $[3,9,10]$.

Toxicity outcomes of ConvRT among women treated with oncoplastic breast conservation surgery has been well reported [11-14]. Maguire et al. showed that at median follow-up of 2.9 years only $6 \%$ of the 79 patients treated with oncoplastic breast surgery followed by ConvRT developed radiation therapy related toxicities [15].

Several large randomized clinical trials with up to 10 year follow-up data compared the HypoRT with ConvRT after breast conservation surgery [11, 16-18]. Results from these trials have shown that disease-free survival and overall survival outcomes are equivalent for both fractionation groups. Moreover, short and long term toxicity profiles were not significantly different between the radiation regimens. As a result of these studies, evidence-based guidelines recommend HypoRT for eligible patients [19]. Hypofractionated whole breast radiotherapy (HypoRT) reduces the duration of treatment with higher dose fractions by treating the entire breast with 2.5-3.20Gy per fraction for a total dose of 39-42.5Gy in 13-16 daily fractions $[11,16-18,20]$. This shorter fractionation schedule has been adapted in modern practice for patients who are $\geq 50$ years old, have Stage pT1-2 pNO diseases, did not receive chemotherapy, and have a radiation dose homogeneity within $\pm 7 \%$ in the central axis plane.

Although the incorporation of oncoplastic surgery into breast conservation therapy decreases re-excision procedures and produces desired cosmetic results, the acute and late toxicity of HypoRT has not been previously described in these patients. Therefore, in this study we compared toxicity outcomes of HypoRT to ConvRT in women treated with oncoplastic breast conservation surgery from a retrospective database. We hypothesized that acute and late toxicity would not be increased with HypoRT compared to ConvRT.

\section{Materials and methods}

We analyzed a series of patients treated with oncoplastic breast conservation surgery followed by HypoRT or ConvRT for early stage breast cancer between January 2008 and December 2014. Inclusion criteria for this study were pathology results that confirmed a diagnosis of early stage (Tis-T2 and $\mathrm{Nx}-1$ ) invasive adenocarcinoma of the breast or ductal carcinoma in situ (DCIS), adaption of the oncoplastic techniques into breast conservation surgery, and planed radiation therapy as a part of the breast conservation therapy. Pre-surgical evaluation included mammography, biopsy, and clinical examination. Surgical management consisted of lumpectomy with sentinel lymph node evaluation immediately followed by oncoplasty for women with invasive breast cancer, and lumpectomy with oncoplasty alone for women with DCIS. Considering that the term of oncoplasty has been used broadly due to its various techniques, in this study, we defined it as any mobilization of the adjacent glandular breast tissue to fill the excision cavity following tumor removal. The majority of oncoplastic reconstructions in our institution are staged after margins are back. Our plastic surgeon defines the procedures to retain principals of maintaining perfusion and support of the nipple areolar complex, obliterating dead space, marking the lumpectomy margins with clips and modifying various reduction pattern techniques to redrape breast tissue and maintain proper contour. Our institutional policy is to offer hypofractionated radiation as a treatment option for women with DCIS or early stage invasive breast cancer (Tis-T2, N0) if the radiation boost is not indicated. Radiation boost to lumpectomy cavity was recommended for patients 60 years or younger, having well-defined lumpectomy cavity, and pathologic features indicating a greater potential benefit, such as a positive margin of resection.

\section{Radiotherapy planning and delivery}

Computed tomography (CT) simulation was completed following the surgery using a Brilliance BigBore CT scanner (Philips Healthcare, Andover, MA). Depending on patient tolerance and anatomy, patient position during simulation was either supine or prone. For supine position, a C-Qual breast board (CIVCO Medical Solutions, Orange City, IA) with an upper alpha cradle was used for positioning, and if the sternal movement was $>0.5 \mathrm{~cm}$, respiratory gating (Varian RPM, Palo Alto, CA) was used. Breast target volume was defined clinically, using standard anatomic boundaries. For the prone setup, patients were positioned using a prone breast board (CDR Systems Inc., Calgary, AB, Canada) further customized for our institution to allow for upper and lower cradle indexing.

In order to optimize coverage and homogeneity, breast plans were created using wedges and large segments or the forward-planned "field in field" technique described by Kestin et al. [21]. In the prone position, only large segments were used for plan optimization to ensure that potential setup variability would not change the intended target coverage. The primary planning goal was to ensure coverage of $95 \%$ of the delineated planning target volume by $>95 \%$ of the prescribed dose (V95 > 95\%). Secondly, plan optimization was performed to minimize heterogeneity by limiting the V105 and V110. Dosimetric parameters were not specifically evaluated at the central axis, rather they were examined based on dose-volume histograms, and dose distributions were reviewed on all axial CT slices. Dose calculations were performed in Pinnacle (Philips Systems, Andover, MA) using the convolution/superposition algorithm to correct for tissue heterogeneities. Treatments were delivered by using a Varian 2100 linear accelerator (Varian Medical Systems, Palo Alto, CA) with 6 and/or higher energy (15 or $18 \mathrm{MV}$ ) photons. The HypoRT dose was 42.56Gy in 2.66Gy per fraction without a boost to the lumpectomy cavity. The ConvRT dose was between 46.80 and 50.40Gy in 1.8 to 2.0Gy per fraction with median boost dose of 14Gy in 2Gy fractions. Boost was used in patients with close or positive margins, with young age, and in the setting of intermediate-high risk features, as described per RTOG 1005 [22].

\section{Data collection and statistical analysis}

Data were reviewed from a retrospectively collected, institutional review board-approved database of patients and was conducted in accordance with the principles of the declaration of Helsinki. All patients provided written informed consent. All data were retrospectively verified 
using the electronic medical records and departmental radiation oncology charts. The clinical parameters of interest included tumor characteristics, stage, age, radiation and surgery techniques, treatment time, acute and late toxicity during radiation and thereafter, and disease control. Acute toxicities included those that occurred during radiotherapy and up to 4 weeks after completion of treatment. The treating radiation oncologist assessed acute toxicity by using institutional skin toxicity reporting as part of the standard weekly on treatment visit and in follow-up examinations [1]. In this skin toxicity report, erythema was scored as a grade 1 toxicity, dry desquamation was scored as a grade 2 toxicity, and focal moist desquamation was scored as a grade 3 toxicity. Treating physicians graded late toxicities using a four-point scale $(0=$ none, $1=$ mild, $2=$ moderate, $3=$ severe).

Statistical analyses were performed with the SPSS statistical software package, version 17.0 (SPSS, Chicago, IL). Univariate analysis was completed to assess associations between treatment position, radiation type, breast volume, and maximum toxicity scores using the test with unequal variance and the chi-square test. P-values $<0.05$ were considered significant.

\section{Results}

Included in this study were 37 women with 38 breasts treated with oncoplastic conservation surgery between 2008 and 2014. One of the woman had bilateral disease, consisting of synchronous tumors. Following the surgery, ConvRT was used for 28 breasts and HypoRT was used for 10 breasts (Table 1). Median follow-up was 15.3 months. Median lag time between surgery and ConvRT was 49 days (range, 33 to 188 days), and 41 days (range, 26 to 52 days) to HypoRT. Median age was 55 years (range, 29 to 69 years) for the ConvRT group and 63 years (range, 58 to 70 years) for the HypoRT group, indicating a significantly younger ConvRT group $(P=0.0073)$. Tumor stage was also significantly different between groups $(P=0.0058)$, with the majority of the ConvRT group consisting of T1 (61\%) and HypoRT with Tis (50\%). Furthermore, nodal status was also significantly different between groups $(P=0.0121)$, with predominant NO (71\%) in the ConvRT group and equal numbers of $\mathrm{Nx}(50 \%)$ and $\mathrm{NO}(50 \%)$ in HypoRT group. Two patients treated with ConvRT had positive margins and the median margin width was $0.3 \mathrm{~cm}$ (range, $0.1-1 \mathrm{~cm}$ ). In HypoRT group all patients had negative margins, with a median margin width of $0.2 \mathrm{~cm}$ (range, $0.04-1 \mathrm{~cm}$ ). There was a significant difference in systemic therapy utilization between groups $(P=0.0457)$. Cytotoxic chemotherapy use was predominant in ConvRT group (64\%), with hormonal therapy utilized in the HypoRT group (70\%). Patients in HypoRT group did not receive chemotherapy. Among the other parameters of interest, there were no significant differences in breast volume or lag time between surgery and radiation in these two groups. In terms of disease control, there were no documented local or distance failures in either group.

All patients treated with HypoRT received 42.56Gy in 2.66Gy fractions (Table 2). A boost was not given in the HypoRT group and regional nodes were not included in the treatment plan. In the ConvRT group, the median dose to
Table 1 Patient characteristics.

\begin{tabular}{|c|c|c|c|}
\hline \multirow{2}{*}{ Characteristics } & \multicolumn{3}{|c|}{ No. treated breasts if applicable } \\
\hline & ConvRT & HypoRT & $P$ value \\
\hline No. patients & 28 & 10 & \\
\hline Age $(y)$ & & & $0.0073^{*}$ \\
\hline Median & 55 & 63 & \\
\hline Range & $(29-69)$ & $(58-70)$ & \\
\hline T Stage & & & $0.0058^{*}$ \\
\hline Tis & $3(11)$ & $5(50)$ & \\
\hline T1 & $17(61)$ & $3(30)$ & \\
\hline $\mathrm{T} 2$ & $7(25)$ & $2(20)$ & \\
\hline T3 & $1(3)$ & $0(0)$ & \\
\hline N Stage & & & $0.0121 *$ \\
\hline $\mathrm{Nx}$ & $2(7)$ & $5(50)$ & \\
\hline No & $20(71)$ & $5(50)$ & \\
\hline N1 & $5(18)$ & $0(0)$ & \\
\hline N2 & $1(3)$ & $0(0)$ & \\
\hline Breast volume $\left(\mathrm{cm}^{3}\right)$ & & & 0.2190 \\
\hline Median & 1022.08 & 907.86 & \\
\hline Range & $\begin{array}{l}(288.11- \\
2117.06)\end{array}$ & $\begin{array}{l}\text { (391.57- } \\
1409.34)\end{array}$ & \\
\hline Systemic therapy & & & $0.0457^{*}$ \\
\hline None & $2(7)$ & $1(10)$ & \\
\hline Tamoxifen/Al & $8(29)$ & $7(70)$ & \\
\hline Cytotoxic chemo & $18(64)$ & $0(0)$ & \\
\hline Lag time (surgery to RT) & & & 0.0741 \\
\hline Median days & 49 & 41 & \\
\hline Range & $(30-188)$ & $(26-52)$ & \\
\hline
\end{tabular}

Abbreviations: ConvRT = conventionally fractionated radiotherapy; HypoRT = hypofractionated radiotherapy; *Statistical significant.

the whole breast was 50.4Gy (range, 46 to $50.4 \mathrm{~Gy}$ ) in $1.8 \mathrm{~Gy}$ fractions. In this group, 16 breasts received an additional boost to the lumpectomy cavity, to a median dose of 10Gy (range, 10 to $16 \mathrm{~Gy}$ ). Furthermore, supraclavicular nodes were radiated with $50.4 \mathrm{~Gy}$ in $1.8 \mathrm{~Gy}$ fractions in 9 patients from the ConvRT group. Four patients received both supraclavicular node radiation and additional boost to the lumpectomy cavity. In the HypoRT group, $70 \%$ of the patients were treated in supine position and $89 \%$ in the ConvRT group ( $P=0.1594)$. There were no statistically significant differences in radiation dosimetric parameters of V95 ( $P=0.5142)$ and V1 $10(P=0.5732)$. However, V105 was significantly increased in ConvRT group (22.5 vs 17.87 , $P=0.0266)$.

Acute skin toxicity was significantly higher among the conventionally treated patients $(P=0.0314)$ (Table 3$)$. The incidence of both grade 2 (dry desquamation) and grade 3 (focal moist desquamation) was significantly higher in 
Table 2 Radiation treatment parameters.

\begin{tabular}{|c|c|c|c|}
\hline Parameters & ConvRT (\%) & HypoRT (\%) & P value \\
\hline Whole breast dose (Gy) & & & NA \\
\hline Median & 50.4 & 42.56 & \\
\hline Range & $(46.00-50.40)$ & $(42.56-42.56)$ & \\
\hline Fraction size (Gy) & & & NA \\
\hline 1.8 & $21(75)$ & $0(0)$ & \\
\hline 2 & $7(35)$ & $0(0)$ & \\
\hline 2.66 & $0(0)$ & $10(100)$ & \\
\hline Boost dose (Gy) & & & NA \\
\hline 0 & $12(43)$ & $10(100)$ & \\
\hline 10 & $10(36)$ & $0(0)$ & \\
\hline 12 & $3(11)$ & $0(0)$ & \\
\hline 14 & $2(7)$ & $0(0)$ & \\
\hline 16 & $1(3)$ & $0(0)$ & \\
\hline \multicolumn{3}{|c|}{ Supraclavicular node dose (cGy) } & NA \\
\hline 0 & $19(68)$ & $0(0)$ & \\
\hline 50 & $1(3)$ & $0(0)$ & \\
\hline 50.4 & $8(29)$ & $0(0)$ & \\
\hline \multicolumn{4}{|l|}{ Position } \\
\hline Prone & $3(11)$ & $3(30)$ & $0.1594 *$ \\
\hline Supine & $25(89)$ & $7(70)$ & \\
\hline \multicolumn{4}{|l|}{ Dosimetric parameters } \\
\hline V95 & 98.54 & 98.57 & 0.5142 \\
\hline V105 & 22.5 & 17.87 & 0.0266 * \\
\hline V110 & 0.1 & 0.2 & 0.5732 \\
\hline
\end{tabular}

Abbreviations: ConvRT = conventionally fractionated radiotherapy; HypoRT = hypofractionated radiotherapy; NA = not available; *Statistical significance.

the ConvRT group, $43 \%$ and $7 \%$, respectively, compared to $10 \%$ and $0 \%$ in HypoRT group. Maximal acute toxicity was experienced during the treatment in both groups. There were no reports of pneumonitis and no differences in acute fatigue or edema. Furthermore, there were no wound dehiscences in either treatment group. For the subset of patients treated conventionally with additional boost $(\mathrm{n}=$ $16)$, there were 10 (62\%) cases of dry desquamation and 2 (13\%) cases of focal moist desquamation. Skin pigmentation was the most commonly reported late skin toxicity, noted by $57 \%$ of the ConvRT and $40 \%$ of the HypoRT patients ( $P$ $=0.163)$. Two (7\%) patients had Grade 2 late skin toxicity in ConvRT group. Two (7\%) patients treated with ConvRT had mild lymphedema versus none in HypoRT group. Late subcutaneous toxicity reported as mild fibrosis was not significantly different the treatment group $(P=0.133)$.

A univariate analysis indicated that breast volume and treatment position were not associated with the development of dry and/or focal moist desquamation
Table 3 Toxicity outcomes (highest grade reported for each patient).

\begin{tabular}{|c|c|c|c|}
\hline & ConvRT (\%) & HypoRT (\%) & Pvalue \\
\hline \multicolumn{4}{|l|}{ Acute toxicity outcomes } \\
\hline Skin toxicity & & & $0.0314 *$ \\
\hline Grade 1 & $14(50)$ & $9(90)$ & \\
\hline Grade 2 & $12(43)$ & $1(10)$ & \\
\hline Grade 3 & $2(7)$ & $0(0)$ & \\
\hline Breast edema & & & 0.5710 \\
\hline Grade 0 & $23(82)$ & $9(90)$ & \\
\hline Grade 1 & $5(18)$ & $1(10)$ & \\
\hline Fatigue & & & 0.1483 \\
\hline Mild & $16(57)$ & $3(30)$ & \\
\hline \multicolumn{4}{|l|}{ Late toxicity outcomes } \\
\hline Skin toxicity & & & 0.1630 \\
\hline Grade 1 & $14(50)$ & $4(40)$ & \\
\hline Grade 2 & $2(7)$ & $0(0)$ & \\
\hline \multicolumn{4}{|c|}{ Subcutaneous tissue toxicity } \\
\hline Grade 1 & $3(11)$ & $2(20)$ & 0.1330 \\
\hline \multicolumn{4}{|l|}{ Lymphedema } \\
\hline Grade 1 & $2(7)$ & $0(0)$ & NA \\
\hline
\end{tabular}

Abbreviations: ConvRT = conventionally fractionated radiotherapy; HypoRT = hypofractionated radiotherapy; *Statistical significance.

(Table 4). The association was seen, however, between maximal acute skin toxicity and radiotherapy techniques (ConvRT vs HypoRT). The mean breast volume $\left(1012.88 \mathrm{~cm}^{3}\right.$ ) experiencing dry and/or focal moist desquamation was not significantly different than the breast volume $\left(1004.89 \mathrm{~cm}^{3}\right)$ experiencing no maximal acute toxicity $(P=0.626)$. The majority of patients (84\%) were treated in supine position and this was not significantly associated with acute toxicity $(P=0.2238)$. Dry and/or focal moist desquamation was seen in a greater number of the conventionally treated women $(93 \%)$ compared to women treated with HypoRT $(7 \%)(P=0.0263)$.

Table 4 Association of clinical factors with acute toxicity.

\begin{tabular}{lccc}
\hline & $\begin{array}{c}\text { Dryand/or focal } \\
\text { moist desquamation }\end{array}$ & $\begin{array}{c}\text { No dry and/or focal } \\
\text { moist desquamation }\end{array}$ & P value \\
\hline $\begin{array}{l}\text { Breast volume } \\
\text { (mean, } \mathrm{cm}^{3} \text { ) }\end{array}$ & 1088.64 & 1004.89 & 0.6260 \\
$\begin{array}{l}\text { Treatment } \\
\text { position (\% } \\
\text { Supine) }\end{array}$ & 43.00 & 57.00 & 0.2238 \\
$\begin{array}{l}\text { Radiotherapy } \\
\text { therapy (\% } \\
\text { HypoRT) }\end{array}$ & 6.70 & & \\
\hline
\end{tabular}

Abbreviations: HypoRT = hypofractionated radiotherapy; *Statistical significance

\section{Discussion}

To the best of our knowledge, this is the first reported study analyzing the acute and late toxicity outcome of 
HypoRT among women treated with oncoplastic breast conservation surgery. The results of our study showed that HypoRT versus ConvRT is not associated with increased acute and late toxicity in this subset of women treated with such new emerging surgical techniques.

We report dry and/or focal moist desquamation in 50\% of the patients treated with ConvRT and $10 \%$ of patients treated with HypoRT. There is no prior reported study to compare our findings from the HypoRT group, and the rate of acute toxicity in conventionally treated women is not similar with the only available previous study [23]. In this recent study, of the 79 patients treated with oncoplastic breast surgery and postoperative radiation therapy, only $6 \%$ of the patients experienced acute toxicity. Conventional radiation therapy with median dose of 46Gy in 1.8-2.0Gy daily fractionation was given with no additional boost to the lumpectomy cavity. In our analysis, the treatment dose was higher (median dose 50.4Gy in 1.8 - 2.0 daily fractionation) and the majority of the women (70\%) treated with ConvRT also received additional boost to the lumpectomy cavity with a median dose of 10Gy in 2.0Gy fractionation. Furthermore, systemic therapy was utilized in $93 \%$ of the ConvRT group in our study versus $58 \%$ in the previous study.

Our data not only supports the hypothesis that acute and late toxicity would not be increased with HypoRT, but also showed that the toxicity rate was lower in this group compared to ConvRT. Many patients treated with radiation therapy develop radiation-induced skin toxicity, with the severity of the reaction associated with treatment related factors such as total dose delivered, use of bolus, and addition of chemotherapy $[15,18]$. ConvRT group was treated with a higher dose of radiation (median dose 50.4Gy) compared to HypoRT group (median dose 42.56Gy), which may be a possible explanation for the higher rates of acute toxicity observed. The additional boost to the lumpectomy cavity (median dose of 10Gy) in this group may also contribute to higher toxicity since $75 \%$ of patients with boost treatment developed acute toxicity. Moreover, $64 \%$ of the patients in ConvRT group received chemotherapy, which may further explain the higher rate of toxicity in this group.

The correlation between breast volume and acute toxicity has been suggested in previous studies [12-14, 24]. In our study, there was no significant difference in breast volume between groups; there was a trend; however, to increased acute toxicity in large breast volume. It has been shown that addition of cytotoxic chemotherapy also leads to an increased incidence of side effect $[25,26]$. Considering $64 \%$ of patients in the ConvRT group received chemotherapy versus $20 \%$ in the HypoRT group, it is possible that addition of chemotherapy in the conventionally treated group contributes to higher rates of acute toxicity.

Computation of biological equivalent dose (BED) can be used to quantify the expected biological effect associated with the treatment of a large fraction dose in a short period. The acutely-reacting normal tissue have a high $\alpha / \beta$ ratio and they are less responsive to change in fraction size. This might further explain why there was no increase in the acute toxicity rate with large fraction dose in HypoRT group compare to ConvRT group. The $\alpha / \beta$ ratios is low in late-reacting normal tissue such as fibroblasts therefore they are very responsive to increase in fraction size, leading to a late fibrosis. This was one of the primary concerns for randomized trials evaluating HypoRT compared to ConvRT, however, the late fibrosis was not worse with larger doses per fraction [11, 16-18]. Furthermore, in this initial report, considering the need for appropriate vascular flow and healing after oncoplasty, our concern that the larger doses used with HypoRT would possibly impact the integrity of the reconstruction was not observed. Considering the late toxicity in HypoRT group was not significantly high, this might be due to the number of patients and duration of the follow-up time. Longer follow-up is needed to confirm our results. However, the follow-up time of just over one year, provides some basis for continuing to assess this and, should there have been increased oncoplasty failure within our small cohort, it would have changed our use of HypoRT with our patients who undergo oncoplasty as part of BCT.

Dose optimization with 3D planning, utilizing positioning, segments, and/or forward-planned field in field techniques was utilized in both treatment groups since these techniques have been shown to reduce acute skin toxicity $[27,28]$. A formal dosimetric comparison between the cohorts was not performed; due to possible confounding factors such as different fraction size and breast dose. The retrospective nature of the analysis in this study may have resulted in selection bias. In order to overcome this, we attempted to include all patients treated with oncoplastic breast conservation surgery followed by either HypoRT or ConvRT in our institution. Limitations of this study may also include the short follow-up time and use of boost in the ConvRT group and failure to control for additional factors associated with toxicity, such as diabetes or smoking.

\section{Conclusion}

The current study shows that HypoRT is not associated with increased toxicity among women treated with oncoplastic breast conservation surgery compared with ConvRT. HypoRT should be explored further as a viable therapeutic option for those patients treated with new emerging surgical techniques, particularly when a boost is not clinically indicated.

\section{Conflicts of interest}

The authors declare no conflicts of interest.

\section{References}

[1] Trotti A, Colevas AD, Setser A, Rusch V, Jaques D, et al. CTCAE v3.0: development of a comprehensive grading system for the adverse effects of cancer treatment. Semin Radiat Oncol. 2003; 13(3):176-181.

[2] van Dongen JA, Voogd AC, Fentiman IS, Legrand C, Sylvester RJ, et al. Long-term results of a randomized trial comparing breast-conserving therapy with mastectomy: European Organization for Research and Treatment of Cancer 10801 trial. J Natl Cancer Inst. 2000; 92(14):11431150.

[3] Fisher B, Anderson S, Bryant J, Margolese RG, Deutsch M, et al. Twentyyear follow-up of a randomized trial comparing total mastectomy, lumpectomy, and lumpectomy plus irradiation for the treatment of invasive breast cancer. N Engl J Med. 2002; 347(16):1233-1241. 
[4] Audretsch WP, Rezai M, Kolotas C. Tumor-specific immediate reconstruction (TSIR) in breast cancer patients. Perspect Plast Surg. 1998; 11:71-100.

[5] Rainsbury RM, Paramanathan N. Recent progress with breastconserving volume replacement using latissimus dorsi miniflaps in UK Patients. Breast Cancer. 1998; 5(2):139-147.

[6] Munhoz AM, Montag E, Arruda EG, Aldrighi C, Gemperli R, et al. The role of the lateral thoracodorsal fasciocutaneous flap in immediate conservative breast surgery reconstruction. Plast Reconstr Surg. 2006; 117(6):1699-1710.

[7] Aljarrah A, Nos C, Nasr R, Clough KB, Bats AS, et al. Updated followup of patients treated with the oncoplastic "Crescent" technique for breast cancer. Breast. 2012; 21(4):475-479.

[8] Clough KB, Kaufman GJ, Nos C, Buccimazza I, Sarfati IM. Improving breast cancer surgery: A classification and quadrant per quadrant atlas for oncoplastic surgery. Ann Surg Oncol. 2010; 17(5):1375-1391.

[9] Veronesi U, Cascinelli N, Mariani L, Greco M, Saccozzi R, et al. Twentyyear follow-up of a randomized study comparing breast-conserving surgery with radical mastectomy for early breast cancer. N Engl J Med. 2002; 347(16):1227-1232.

[10] Overgaard M, Hansen PS, Overgaard J, Rose C, Andersson M, et al. Postoperative radiotherapy in high-risk premenopausal women with breast cancer who receive adjuvant chemotherapy. Danish Breast Cancer Cooperative Group 82b Trial. N Engl J Med. 1997; 337(14):949955.

[11] Bentzen SM, Agrawal RK, Aird EG, Barrett JM, Barrett-Lee PJ, et al. The UK Standardisation of Breast Radiotherapy (START) Trial B of radiotherapy hypofractionation for treatment of early breast cancer: a randomised trial. Lancet. 2008; 371(9618):1098-1107.

[12] Back M, Guerrieri M, Wratten C, Steigler A. Impact of radiation therapy on acute toxicity in breast conservation therapy for early breast cancer. Clin Oncol (R Coll Radiol). 2004; 16(1):12-16.

[13] Fernando IN, Ford HT, Powles TJ, Ashley S, Glees JP, et al. Factors affecting acute skin toxicity in patients having breast irradiation after conservative surgery: a prospective study of treatment practice at the Royal Marsden Hospital. Clin Oncol (R Coll Radiol). 1996; 8(4):226-233.

[14] Moody AM, Mayles WP, Bliss JM, A'Hern RP, Owen JR, et al. The influence of breast size on late radiation effects and association with radiotherapy dose inhomogeneity. Radiother Oncol. 1994; 33(2):106112.

[15] Bentzen SM, Turesson I, Thames HD. Fractionation sensitivity and latency of telangiectasia after postmastectomy radiotherapy: a gradedresponse analysis. Radiother Oncol. 1990; 18(2):95-106.

[16] Whelan T, MacKenzie R, Julian J, Levine M, Shelley W, et al. Randomized trial of breast irradiation schedules after lumpectomy for women with lymph node-negative breast cancer. J Natl Cancer Inst. 2002; 94(15):1143-1150.

[17] Bentzen SM, Agrawal RK, Aird EG, Barrett JM, Barrett-Lee PJ, et al. The UK Standardisation of Breast Radiotherapy (START) Trial A of radiotherapy hypofractionation for treatment of early breast cancer: a randomised trial. Lancet Oncol. 2008; 9(4):331-341.

[18] Whelan TJ, Pignol JP, Levine MN, Julian JA, MacKenzie R, et al. Long-term results of hypofractionated radiation therapy for breast cancer. N Engl J Med. 2010; 362(6):513-520.

[19] Smith BD, Bentzen SM, Correa CR, Hahn CA, Hardenbergh PH, et al. Fractionation for whole breast irradiation: an American Society for Radiation Oncology (ASTRO) evidence-based guideline. Int J Radiat Oncol Biol Phys. 2011; 81(1):59-68.

[20] Owen JR, Ashton A, Bliss JM, Homewood J, Harper $C_{1}$ et al. Effect of radiotherapy fraction size on tumour control in patients with earlystage breast cancer after local tumour excision: long-term results of a randomised trial. Lancet Oncol. 2006; 7(6):467-471.

[21] Kestin LL, Sharpe MB, Frazier RC, Vicini FA, Yan D, et al. Intensity modulation to improve dose uniformity with tangential breast radiotherapy: initial clinical experience. Int J Radiat Oncol Biol Phys. 2000; 48(5):1559-1568

[22] Radiation Therapy Oncology Group. RTOG 1005 Protocol information. 2014.

[23] Maguire PD, Adams A, Nichols MA. Oncoplastic surgery and radiation therapy for breast conservation: early outcomes. Am J Clin Oncol. 2015; 38(4):353-357.

[24] Corbin KS, Dorn PL, Jain SK, Al-Hallaq HA, Hasan Y, et al. Hypofractionated radiotherapy does not increase acute toxicity in large-breasted women: results from a prospectively collected series. Am J Clin Oncol. 2014; 37(4):322-326.
[25] Fiets WE, van Helvoirt RP, Nortier JW, van der Tweel I, Struikmans H. Acute toxicity of concurrent adjuvant radiotherapy and chemotherapy (CMF or AC) in breast cancer patients. A prospective, comparative, non-randomised study. Eur J Cancer. 2003; 39(8):1081-1088.

[26] Hanna YM, Baglan KL, Stromberg JS, Vicini FA, A Decker D. Acute and subacute toxicity associated with concurrent adjuvant radiation therapy and paclitaxel in primary breast cancer therapy. Breast J. 2002; 8(3):149-153.

[27] Pignol JP, Olivotto I, Rakovitch E, Gardner S, Sixel K, et al. A multicenter randomized trial of breast intensity-modulated radiation therapy to reduce acute radiation dermatitis. J Clin Oncol. 2008; 26(13):20852092

[28] Donovan E, Bleakley N, Denholm E, Evans P, Gothard L, et al. Randomised trial of standard 2D radiotherapy (RT) versus intensity modulated radiotherapy (IMRT) in patients prescribed breast radiotherapy. Radiother Oncol. 2007; 82(3):254-264. 